NISTIR 7746

\title{
WSQ Problem with Two-Thumb Captures from Large Platen Live-Scan Devices
}

Craig Watson 
NISTIR 7746

\title{
WSQ Problem with Two-Thumb Captures from Large Platen Live-Scan Devices
}

\author{
Craig Watson \\ Information Technology Laboratory \\ Information Access Division
}

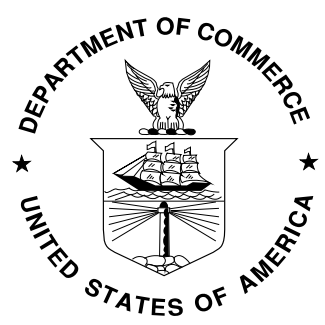

U.S. Department of Commerce 


\title{
WSQ Problem with Two-Thumb Captures from Large Platen Live-Scan Devices
}

\begin{abstract}
This paper investigates an issue with the Wavelet Scalar Quantization (WSQ) compression algorithm [1] which causes severe degradation of the compressed image. The problem was first noticed when compressing the two-thumb images from live-scan identification flats (ID Flats) captured with a 3-inch high platen device. If the thumbs were placed sufficiently close to the bottom edge of the platen during capture, the compression/decompression of the image could result in a severely degraded fingerprint image. In extreme cases all fingerprints in the image were lost. NIST testing on 3-inch identification slaps shows $2 \%-3 \%$ of the two-thumb captured images are affected by some level of degradation. This paper will explain why this occurs, show an example, and offer an algorithm modification to detect and prevent the problem. It is recommended that modifications to the WSQ specification and existing operational software be made to prevent future degradation to $2 \%-3 \%$ of two-thumb compressed images that are currently going undetected in automated capture systems.
\end{abstract}

\section{Introduction}

The WSQ algorithm is widely used in law enforcement and civilian applications for the compression of 8-bit gray-scale fingerprint images captured at 500 pixels per inch (ppi, $19.7 \mathrm{ppmm}$ ) resolution. Image compression reduces the number of bytes needed to digitally store and transmit fingerprint images. The WSQ specification [1] defines parameters used by the WSQ compression algorithm to maintain a desired level of data entropy such that the reconstructed fingerprint image remains useful for fingerprint verification/identification and for forensic comparison applications. On average, WSQ compression of rolled fingerprints as defined in the specification results in a compression ratio around 15:1.

The WSQ algorithm performs a decomposition of the fingerprint image into 64 subbands using a discrete wavelet transform (DWT). Each subband contains information about the fingerprint image in a particular frequency range. Image compression is achieved by quantizing each subband at varying amounts and then Huffman encoding the quantized values. Information is lost during the WSQ compression process so the reconstructed image is not exactly the same as the original fingerprint image. Studies previously performed [3] show which frequency subbands are most important to reconstructing a fingerprint image closest in quality to the original fingerprint image. The level of quantization for each subband is based in part on a variance computation for that subband. The variance computation is based on a subregion of the subband and it is at this stage where the problem occurs with two-thumb images captured on 3-inch platen live-scan devices.

It should be noted that WSQ compression has been successfully used since 1993 for both live-scan and digitally scanned ink impressions. WSQ has been extremely successful at reducing the amount of data storage needed to maintain large databases of digitized fingerprint images. It is only with the recent 
addition in technology of 3-inch high live-scan slap capture devices that the problem with the compression algorithm has occurred.

\section{WSQ Subband Variance Computation}

Image degradation during WSQ compression is caused by the subregion used to compute the subband variance. The current WSQ specification requires the variance to be computed for each of the 64 wavelet subbands using a subregion of that subband as per the following formulas:

$$
\begin{aligned}
& \text { variance_x_start_point }=1 / 8 * \text { subband_width } \\
& \text { variance_y_start_point }=9 / 32 * \text { subband_height } \\
& \text { variance_width }=3 / 4 * \text { subband_width } \\
& \text { variance_height }=7 / 16 * \text { subband_height }
\end{aligned}
$$

Figure 1 shows the wavelet subbands from the WSQ specification and a representative subband with the subregion used for variance computation highlighted with a dashed line. This variance computation works for digitally scanned ink cards, individually captured live-scan images, 2-inch platen live-scan slap capture devices and the majority of 3-inch live-scan four-finger slap captures. It is possible for the twothumb image captured on 3-inch platen devices, to have very little fingerprint ridge structure inside the variance computation subregion resulting in an underestimation of the subband variance. This underestimation of the variance can result in severe degradation or complete loss of the fingerprint information during image compression. Figure 2 and Figure 3 shows an example of a two thumb impression with the images located near the bottom of the platen. Captures like this are subject to degradation during compression as shown in the reconstructed image in Figure 2 and Figure 3.
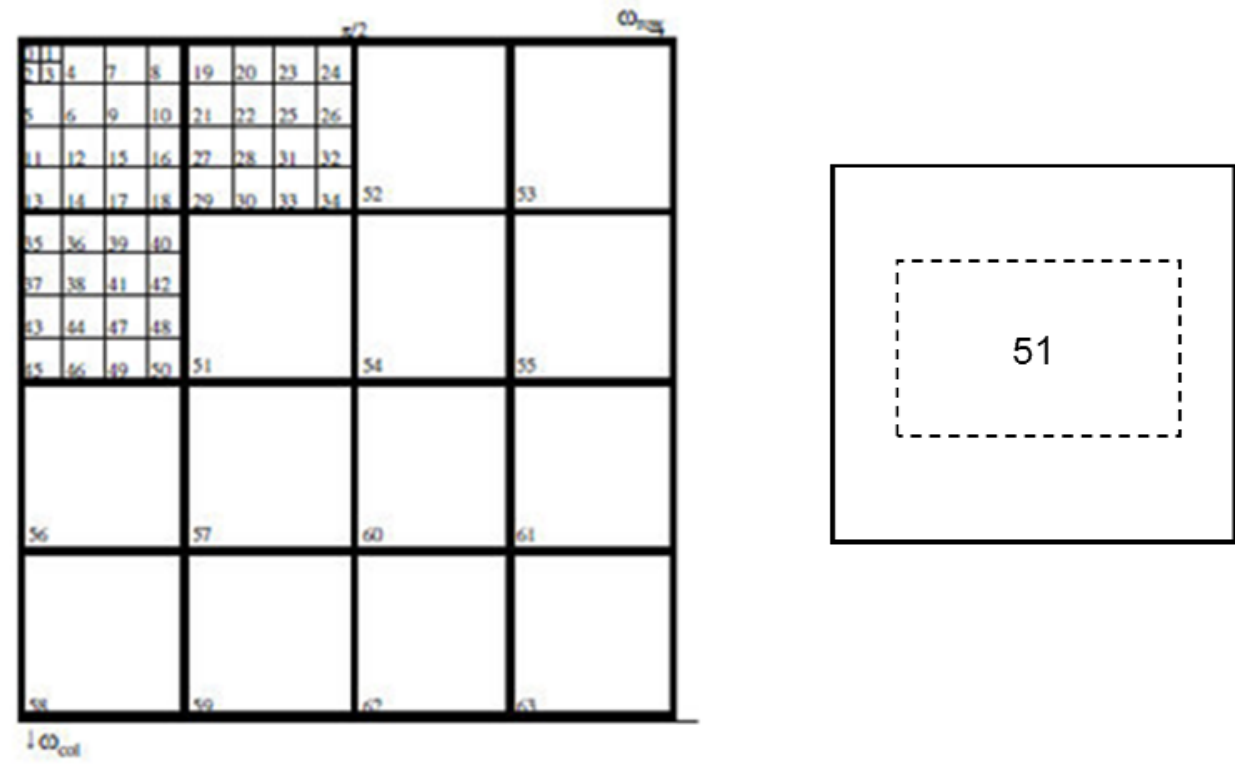

Figure 1. Wavelet subbands and a representative subband with dashed line defining the subregion within which the variance is computed. 


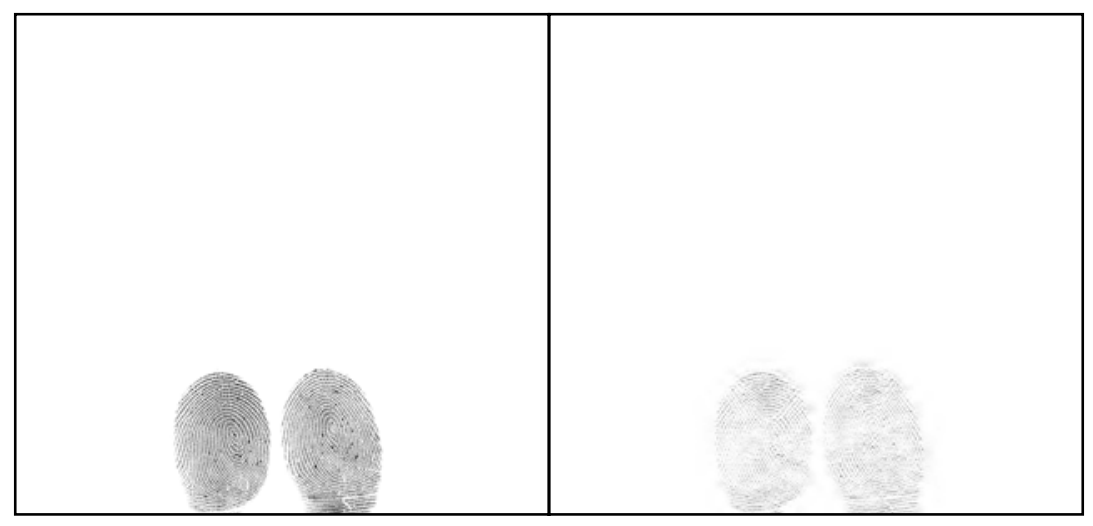

Figure 2. Original and reconstructed image showing degradation.
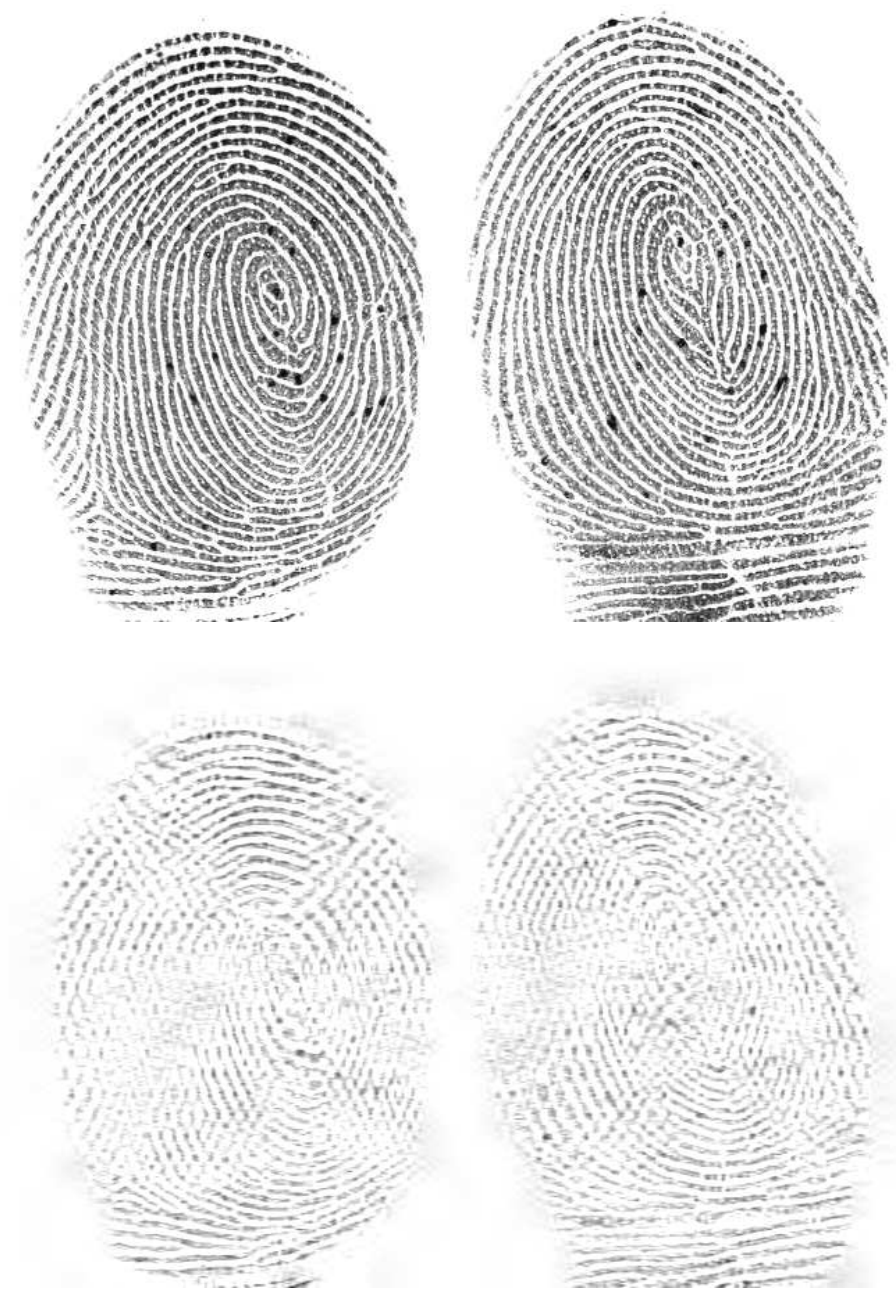

Figure 3. Images from Figure 2 with fingerprint ridge area magnified. 


\section{Modifications to WSQ Subband Variance Computation}

The issue this modification addresses is fingerprints outside the subregion used for variance computation leading to a computed variance that doesn't properly represent the fingerprint data in the image. The bad variance leads to poorly informed quantization decisions that cause irreversible corruption to the fingerprint image. The goal of this modification is to improve the variance computation as needed. The modification is accomplished in two steps. First, a testing condition is used to determine if the subregion variance is unrepresentative of the fingerprint data. Then, when needed the variance is computed over the full region so the fingerprint data is properly represented during quantization.

The WSQ algorithm modification is isolated to part 3-section 3.1 "Subband variance computation" on page 44 of the WSQ specification [1]. The current subband variance is computed based on a subregion of each subband as previously described in section 2 of this document and part3-section 3.1 in the WSQ specification. The new method for subband variance computation makes a decision to use either the subregion or full region of the subband based on the sum of the subregion variance estimates for DWT subbands $0-3$ as shown in Figure $1.1 \sum_{k=0}^{3} \sigma_{k}^{2}>20,000 \mathrm{f}$, the variance computation is based on the subregion of each DWT subband, otherwise the variance computation uses the full region of each DWT subband.

The threshold of 20,000 was selected by analyzing two-thumb captures from an operational system as well as several thousand synthesized two-thumb captures (see Figure 2) designed to create severe compression degradation. The "synthesized" data used two single finger captures from NIST Special Database 24 [7] and started with the images just outside the subregion variance computation area. Then the images were shifted numerous times up/ left/right ( 5 pixels each time) to create samples to test along the subregion variance computation boundary. The image was padded with white pixels as needed to create the same geometry image as the operational data captured on a 3-inch platen. In addition, approximately 1 million images from other operational datasets were used to test the effect of the threshold on existing images.

Finally, the NIST Spectral Image Validation/Verification Utility (SIVV) [8] was used to plot the power spectrum for the original image, WSQ, and WSQ-new for several synthesized two-thumb cases. Figure 4 shows the power spectrum plot for the first synthesized case that didn't result in a complete loss of the prints upon reconstruction (see Figure 3). Notice the significant improvement in the power spectrum of WSQ-new compared to WSQ and the "Original" uncompressed image. Figure 5-Figure 8 show the power spectrum plots as the images were shifted upward and resulted in various threshold points of the summed variances for subbands 0-3. Figure 5-Figure 8 also have a second plot with only the right tail of the spectrum visible. The variation from 10,000 to 20,000 was very small on the right of the spectrum but being conservative it made sense to use the 20,000 threshold point. This was supported by visually examining operational data that showed degraded images with summed variances in the 10,000-15,000 range. Notice that at the threshold of 20,000 the WSQ and WSQ_new are performing almost the same. 


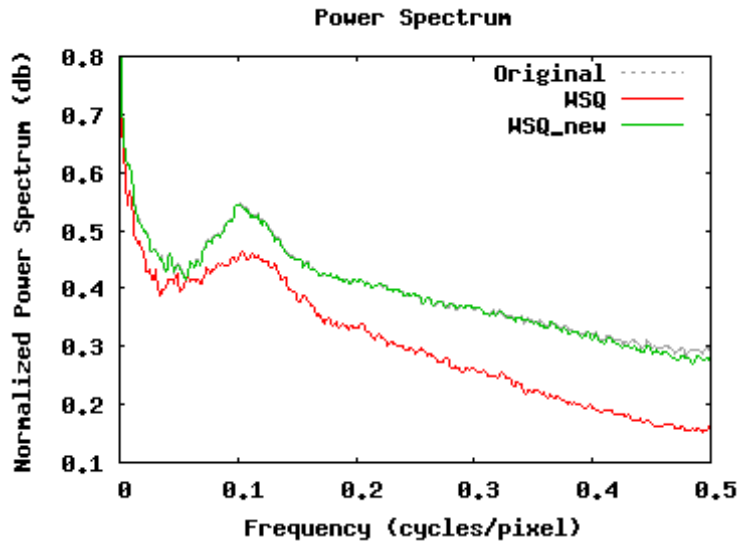

Figure 4. Power spectrum plot from SIVV for severely degraded image in Figure 2.
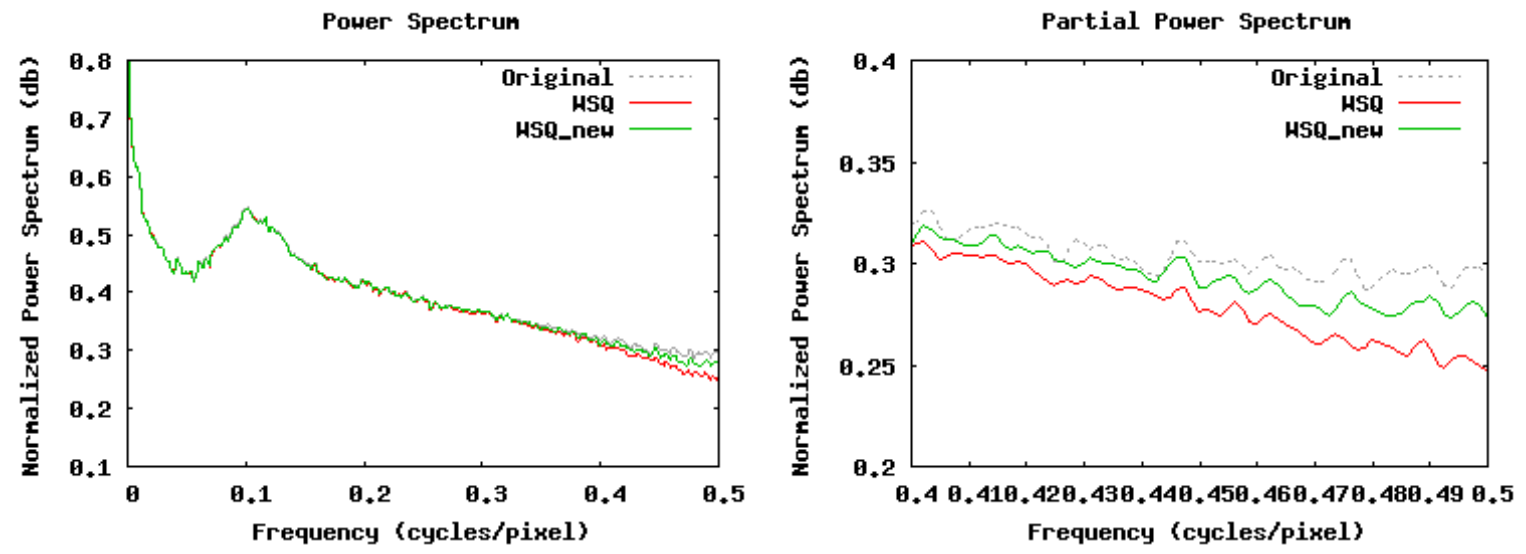

Figure 5. Power spectrum when $\sum_{k=0}^{3} \sigma_{k}^{2} \approx 1,000$. 

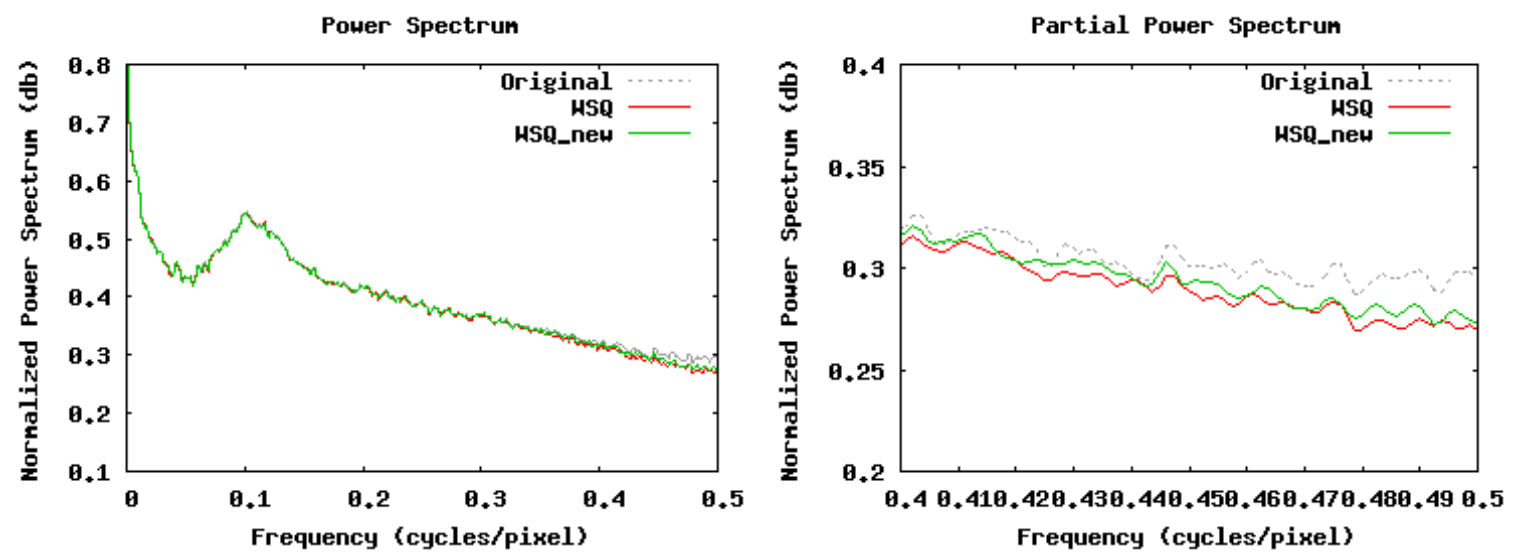

Figure 6. Power spectrum when $\sum_{k=0}^{3} \sigma_{k}^{2} \approx 5,000$.
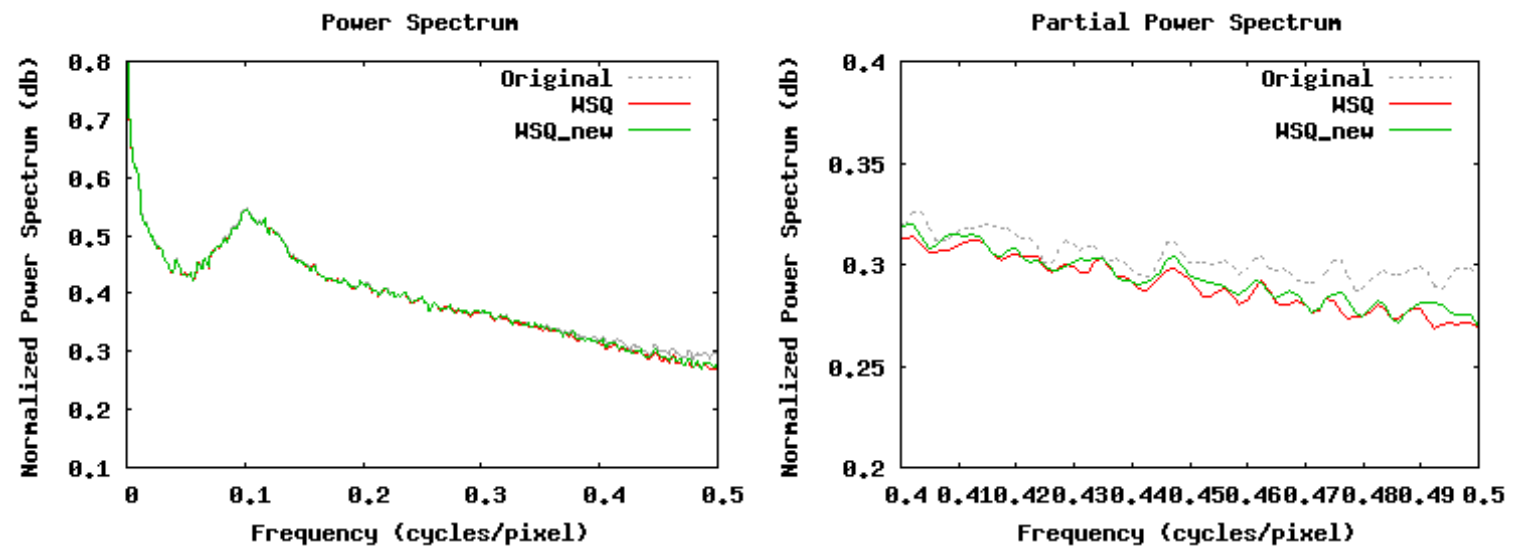

Figure 7. Power spectrum when $\sum_{k=0}^{3} \sigma_{k}^{2} \approx 10,000$.
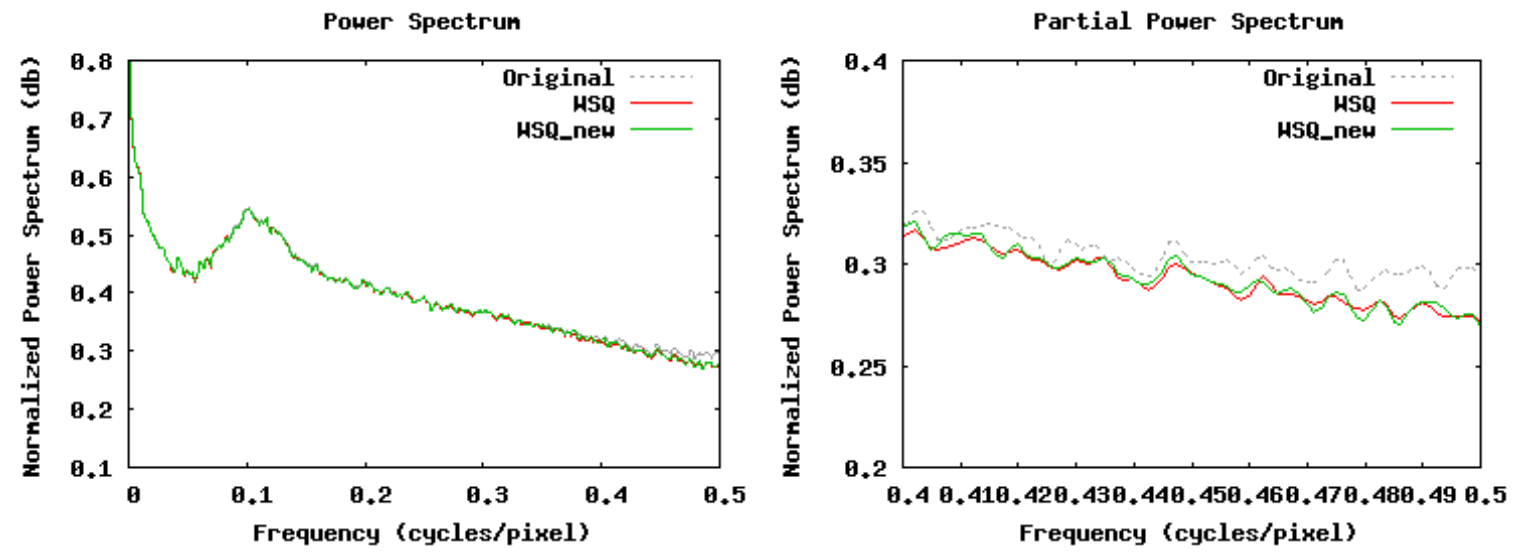

Figure 8. Power spectrum when $\sum_{k=0}^{3} \sigma_{k}^{2} \approx 20,000$.

Finally, a visual inspection was performed on some of the images looking at fine detail like sweat pores. Figure 9 shows the top image from Figure 3 with a box showing the area magnified in Figure 10 for 
both the original image, WSQ-new reconstructed, and WSQ reconstructed. Figure 11 shows the same magnified areas for the images when
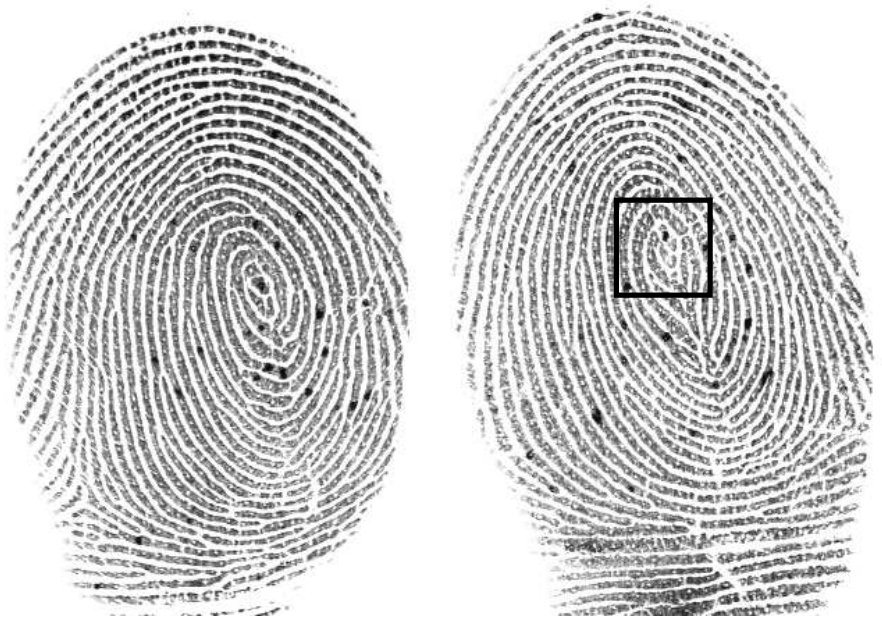

Figure 9. Top image from Figure 3 with box showing area magnified in Figure 10.
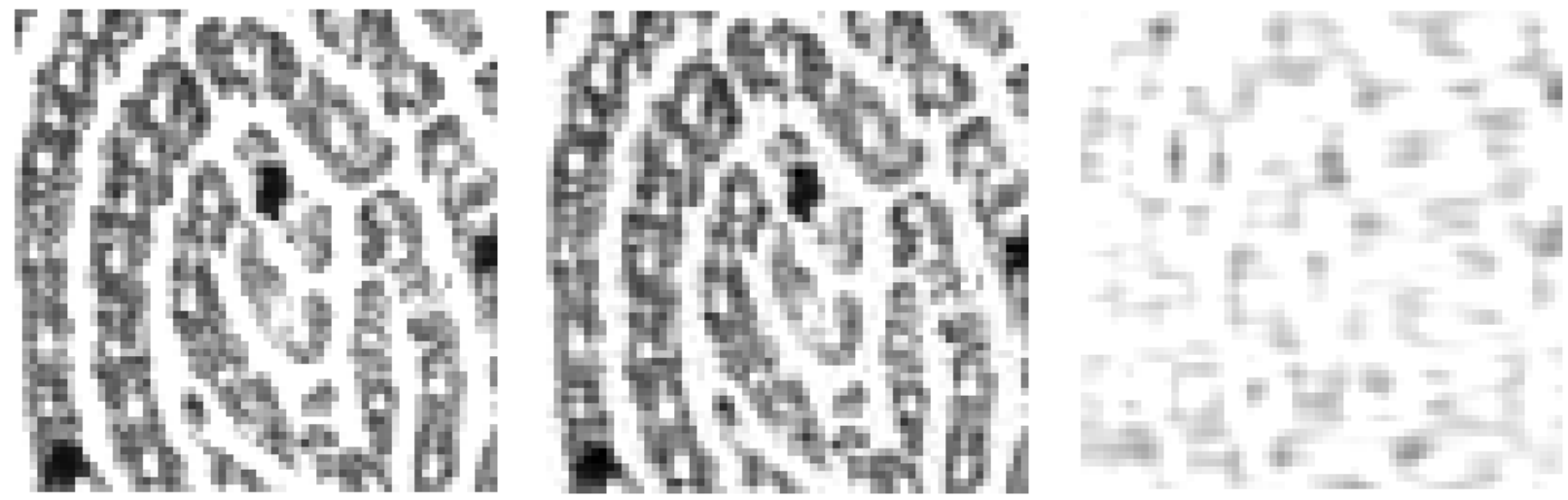

Figure 10. Magnified area for original (left), WSQ-new (center), and WSQ (right).
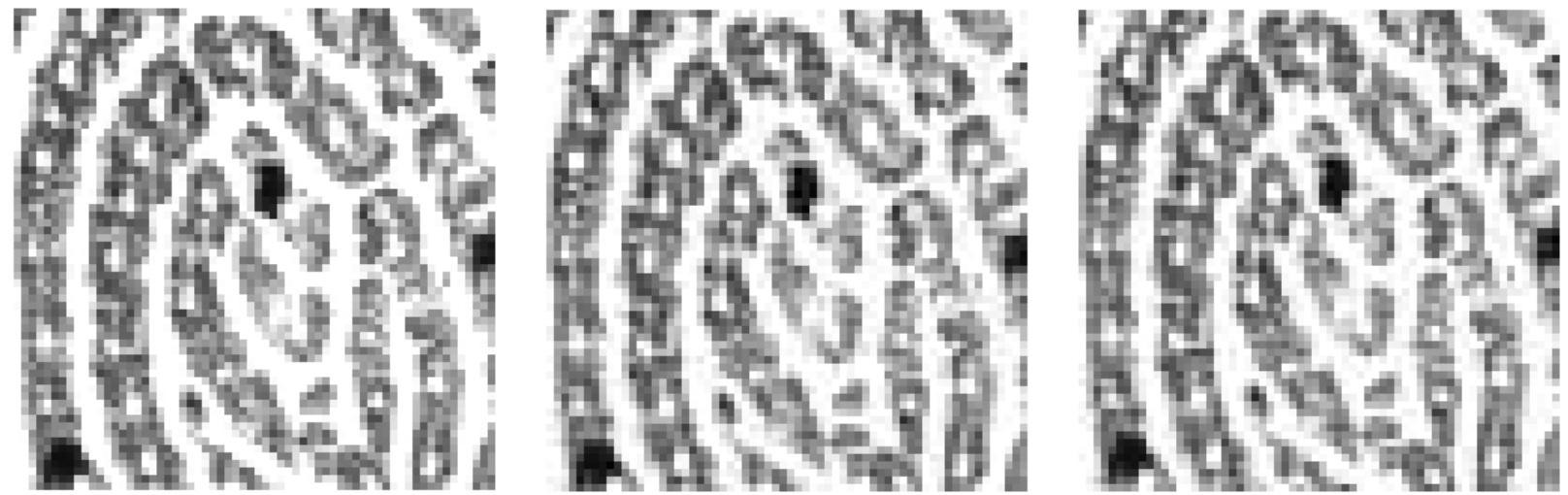

Figure 11. Magnified area for original, WSQ-new, and WSQ when 


\section{Conclusions}

The algorithm modification presented in this paper only alters the variance computation on an as needed basis and mainly for larger platen live-scan capture of two-thumb images. It is slightly possible that single finger captures and slap images captured on platens smaller than 3 inches in height will use this new variance computation when compressed. Studies done on existing single finger live-scan, rolled image, and Identification Flat datasets showed that a small percentage of non two-thumb captures were affected by this change. Compression analysis of 500,000 single finger live-scan capture impressions and 100,000 four-finger slap capture impressions showed that $<0.1 \%$ and $<0.5 \%$ of each image type respectively did undergo full-region variance estimation as a result of this modification. Visual inspection of both types showed that the affected images were generally low quality with very little visible ridge structure. Based on compression analysis of 330,000 two-thumb impressions this modification affected approximately $12 \%$ of these impressions captured on a large platen live-scan device. The code modification specifically targeted cases where the subregion variance computation showed little to no variance. The modification also protects other applications that use a different capture device which allow most of the image to be captured outside the subregion variance computation area.

Operationally, very little change occurs for images other than two-thumb captures. Since the variance computation decision was based on the subregion variances for subbands $0-3$, the compression time for the subregion variance method was practically identical to the previous algorithm. There was approximately a $20 \%$ increase in compression time for the $12 \%$ of two-thumb captures that used full region variance computation.

The reason for processing $12 \%$ of the two-thumb captures was that in order to get all of the $2-3 \%$ that were obviously affected by the variance problem some non-affected images were also under the summed variance threshold of 20,000. There was not a perfect threshold that flagged everything under threshold as definitely bad and everything over as definitely good. Nothing indicated that full region variance was detrimental to any of the live-scan image captures so it was decided to err on the side of caution and be very confident that everything over the summed variance threshold was good. It was also a timing issue. Since full sub-band variance takes about $20 \%$ longer to compress, some care was taken to not waste time on full region variance compression when it wasn't needed. The final threshold was chosen based on analyzing the problem images and then checked to see how much of the operational data was affected. In the end, it took processing $12 \%$ of all the two-thumb captures with full region variance in order to insure the $2 \%-3 \%$ of degraded were found and corrected.

\section{Recommendations}

There is a clear need to address the degradation caused by the WSQ compression algorithm in cases where the two-thumb impressions are captured near the bottom edge of a large platen surface. Initial analysis shows that around $2 \%-3 \%$ of these impressions are currently being affected by this problem. In extreme cases, the fingerprints are completely deleted from the compressed image. A simple solution 
is proposed to eliminate this loss of information and without this change the use of these images for forensic purposes is either diminished or completely lost. This modification changes only the variance computation in the compression algorithm. The decompression algorithm remains the same and can decompress images from either version of the compression algorithm.

It is recommended that operational systems make this change to prevent future irrecoverable loss/degradation of two-thumb images. The change has been made to the NIST WSQ reference implementation that is part of NBIS release 3.3.1 and is available at http://www.nist.gov/itl/iad/ig/nigos.cfm.

The WSQ specification should be modified to reflect this modification and when ready be made available for downloaded at https://www.fbibiospecs.org/biometric specs.html.

Future WSQ algorithms should be checked during compliance testing (http://www.nist.gov/itl/iad/ig/wsq compliant.cfm) to confirm this modification has been implemented. How to deal with previously certified algorithms appearing at https://www.fbibiospecs.org/wsq/Implementations/Default.aspx is still being discussed. 


\section{References}

1. "WSQ Gray-scale Fingerprint Compression Specification", Criminal Justice Information Services, IAIFS-IC-0110(V3), December 19, 1997. Available at https://www.fbibiospecs.org/biometric specs.html

2. M. Antonini, M. Barlaud, P. Mathieu and I. Daubechies, "Image Coding Using Wavelet Transform", IEEE Trans. Image Processing, vol. 1, pp. 205-220, April 1992.

3. J. N. Bradley, C. M. Brislawn, "1992 Progress Report: Compression of Fingerprint Data Using the Wavelet Vector Quantization Image Compression Algorithms", Los Alamos National Laboratory, April, 1992, Tech. Report \#LA-UR-92-1507.

4. M. Brislawn, "Classification of Symmetric Wavelet Transforms", Los Alamos National Laboratory, Aug. 1992, Tech. Report \#LA-UR-92-2823.

5. Data Format For The Interchange of Fingerprint Information, American National Standard, ANSI/NBS-CLS 1-1993.

6. T. Hopper, F. Preston, "Compression of Gray-Scale Fingerprint Images," in Proc. Data Compression Conference, Snowbird, Utah, March 24-27, 1992, pp. 309-318.

7. NIST Special Database 24 - Digital Video of Live-Scan Fingerprint Data, July 1998. Available at http://www.nist.gov/srd/nistsd24.cfm

8. NIST Spectral Image Validation/Verification Utility (SIVV) is part of the NIST Biometric Image Software (version 3.3.1) which is available for download at http://www.nist.gov/itl/iad/ig/nigos.cfm 